\title{
Esports and the Platforming of Child's Play During COVID-19
}

\author{
Valerie Verdoodt ${ }^{a}$, Robbie Fordyce ${ }^{b}$, Lisa Archbold ${ }^{c}$, Faith Gordon ${ }^{d}$, \\ Damian Clifford $^{e}$
}

a) LSE Department of Law, The London School of Economics and Political Science, London, UK v.verdoodt@lse.ac.uk b) School of Media, Film, and Journalism, Monash University, Melbourne, Melbourne, Australia robbie.fordyce@monash.edu c) Melbourne Law School, University of Melbourne, Melbourne, Australia l.archbold@unimelb.edu.au

d) ANU College of Law, Australian National University, Canberra, Australia faith.gordon@anu.edu.au

e) ANU College of Law, Australian National University, Canberra, Australia damian.clifford@anu.edu.au

\begin{abstract}
The coronavirus pandemic has significantly restricted children's opportunities for play and socialisation with friends in physical outside spaces. As a consequence, children's participation in educational, play and entertainment activities are now predominantly taking place online. One form of online play which has become immensely popular with a young audience, is esports. However, esports and the platforming of play have been associated with public health concerns and excessive commercialisation. This paper will therefore address these issues through the lens of children's rights, in particular the right to play and the right to protection from exploitation. It will explore whether esports can contribute to the realisation of the right to play and enable other rights such as the right to development, assembly and freedom of expression during COVID-19.
\end{abstract}




\section{Keywords}

esports - COVID-19 - article 31 CRC - play - commercialisation - harm - exploitation

\section{Introduction}

The Secretary General of the UN, António Guterres, has suggested that the coronavirus pandemic is quickly turning into a 'broader child rights crisis' (UN News, 202O). It is shining a light on confronting issues, which have previously been hidden and unaddressed. In light of lockdowns, the closure of schools and playgrounds has resulted in unprecedented restrictions being placed on children's access to physical outside spaces for play. Children's participation in educational activities, play, socialisation with their peers and entertainment are now predominantly taking place online. Although the online world opens important new avenues for children to exercise their rights, including their right to play, there are growing concerns about the emergence of new or hidden forms of exploitation and harm - emotionally, psychologically and financially.

In recent years, there has been a rapid increase in the popularity of "Esports" or competitive video game contests (Gibbs et al., 2018). This phenomenon presents significant public health concerns linked to the associated excessive gaming consumption as well as potentially exposing children to harms similar to those in traditional sporting contexts, due to team environments and professional coaching (ESIC, 2019). Moreover, the blurring lines between esports gaming and esports gambling (Zendle, 202O) and the manipulative effect of personalised commercial content embedded as part of the gaming content (Verdoodt et al., 2016) are also of key concern. This commercialisation of play leads to some fundamental questions. For instance, could the playing or watching of esports fall under the concept of "play" or "recreation" as conceptualised by the United Nations Convention on the Rights of the Child ("CRC") and, hence, contribute to the realisation of the rights under Article $31 \mathrm{CRC}$ and enable other rights such as the rights to development, assembly and freedom of expression during COVID-19? The platforming of child's play, therefore, raises important questions from a children's rights perspective.

When responding to such questions and the concerns raised by developments such as esports, it is important, however, not to 'disproportionately restrict children's rights to participation and play' (UNICEF, 2O19), in 
particular in times of Covid-19. This paper will therefore focus on the digitalisation and platforming of child's play during the pandemic and will address the issues through the lens of children's rights, in particular the right to play (Article $3^{1} \mathrm{CRC}$ ) and the right to protection from exploitation (Articles 32 and $36 \mathrm{CRC}$ ). The analysis proceeds in three parts. The first introduces children's rights and esports, the second narrows down on how playing esports can contribute to the realisation of the rights enshrined in Article 31 CRC in times of COVID-19, and the final section explores exploitative play and the need for a balanced regulatory response to the challenges posed by the esports phenomenon.

\section{2 \\ Children's Rights and Esports}

Children are awarded a right to culture, leisure and play under Article $3^{1}$ of the CRC. These elements are interlinked and together are considered essential conditions for the well-being and development of children. The United Nations Committee on the Rights of the Child ("CRC Committee") stresses that the realisation of these elements is 'fundamental to the quality of childhood, to children's entitlement to optimum development, to the promotion of resilience and to the realisation of other rights' (CRC Committee, 2013). The right to play facilitates creativity, imagination and self-confidence. As children learn by doing, play contributes to all aspects of their learning and education. Playful experiences often involve social engagement, allowing children to practise their negotiating skills and resolve conflicts and, as such, they are a form of participation in everyday life (Zosh et al., 2017).

Children are highly engaged users of information and telecommunications technologies, with one third of internet users being younger than 18 years old (Livingstone et al., 2016). The digitalisation of children's "lifeworlds" significantly influences not only how they can exercise their rights, but also how these rights may be supported or neglected (Third et al., 2014). Children's rights have digital dimensions and as such the principles and provisions of the children's rights framework should be looked at through a digital lens (Lievens et al., 2018). Within this context, the digital environment offers great opportunities for the realisation and promotion of the Article 31 rights (CRC Committee, 2021).

\subsection{Play Before and During coviD-19}

Playing online games has become one of the most popular indoor activities, with research showing, for instance, that two in three children report playing 
online games at least once a week in most EU countries (Smahel et al., 2020). The CRC Committee has highlighted that -

children in all regions of the world are spending increasing periods of time engaged in play, recreational, cultural and artistic activities, both as consumers and creators, via various digital platforms and media.

(CRC COMMITTEE, 2013)

In 2015, a study by the OECD showed that an average of 34 per cent of adolescents reported playing videogames every day or almost every day (OECD, 2017). As emerging research is illustrating, this trend has only intensified during the pandemic, as opportunities for exercising the rights under Article 31 CRC have been heavily restricted (Gordon, 2021). For example, a study of the experiences of Australian teens in September 2020 found that they were spending a weekly average of 14.4 hours online, and that 77 per cent of teens spent time online playing games with others (eSafety Commissioner, 2021). The closure of schools, day-care centres, public playgrounds and other arenas for children to play and spend their leisure time such as football fields and art centres caused children to turn to the digital environment for their entertainment. Indeed, emerging qualitative research such as Gordon's, which was conducted during lockdown in the UK with children, young people and professionals, illustrates the notable increase in usage of gaming platforms, particularly by boys and young men (Gordon, 2021).

One form of gaming that has achieved mainstream popularity in recent years are "esports" or competitive video game contests. Pre-covid, esports were already a major category of social/online videogaming, allowing diverse interactions between young people including as both a form of play and as a form of spectatorship. Esports also acted as a major context for online socialisation across platforms as diverse as YouTube, WeChat, Facebook, Twitter and Discord. One major platform for esports is Twitch. As the major market-leading dedicated livestreaming platform for videogames, Twitch can serve as a barometer for changes in video game content engagement during the pandemic. From the outset of the Covid-19 pandemic, emerging reports indicate that video game usage during peak hours has gone up 75 per cent in countries such as the US (Verizon, 2020). During 2020, Twitch showed an 83 per cent growth in hours watched, from 9 billion hours in 2019 to 17 billion in 2020 (Streamelements, 2021). This is happening at a time where there has been generally less time, opportunities and facilities for other (playful) activities. Indeed, with children engaging more frequently in the esports 
world, both their mental capacity and physical time to play offline potentially reduces and these activities represent a more significant part of their lived experience.

On the one hand, esports could offer interesting opportunities for children to alleviate lockdown boredom and stress, and to find new ways to interact with others in times of social distancing. Significantly, emerging qualitative research also demonstrates that they use gaming platforms as part of their socialisation and that for many it fosters a sense of "community" and "belonging" (Gordon, 2021). Even the World Health Organisation ("WHO") has endorsed the playing of videogames during the pandemic in its \#PlayApartTogether campaign and relied on the videogames industry to disseminate messages to help slow the spread of COVID-19 (Businesswire, 2020). This is despite the fact that the WHO voted to include video game addiction or "gaming disorder", in its International Classification of Diseases in 2019 (wHO, 2019).

As will be explored in more detail below, the esports world more specifically has also been associated with significant risks to children's mental health and well-being, due to the use of potentially addictive game elements modelled on gambling, highly competitive and potentially "toxic" play cultures, and their profoundly commercialised nature. Indeed, children's increased engagement in esports during the pandemic also entails that large amounts of data on their preferences, abilities, skills, strengths and weaknesses are being recorded, which can later be reused for commercial purposes (Lopes-Cabarcos et al., 2020 ). But what does the term "esports" actually mean and what activities are captured by this categorisation?

\subsection{The Esports Phenomenon}

"Esports" is a term that describes the forms of play and social organisation that emerge when the organisational principles of traditional sports are applied to video game contexts and culture. Or, more succinctly, what happens when you treat videogaming as a sport. The most publicly-visible aspect of esports are the massive-attended tournaments of multiplayer competitive videogames. Branded teams with significant sponsorship contest these games in large convention centres or stadiums, with the games themselves broadcast online over dedicated platforms. These competitions, many of which are annual and can last several weeks, may focus on one or many games and easily attract in-person crowds of tens of thousands. The largest recent in-person competition, Intel Extreme Masters XIII, in 2019, reportedly attracted some 174,000 in-person attendees, with as many as 1.3 million additional viewers online (Sznajder, 2019). While these attendance numbers are difficult to verify, independent 
observers suggest similar numbers for this same event: approximately 1.2 million viewers (escharts.com, 2019). These competitions are major events, focusing on the most popular of titles, and supported by some of the largest companies in the technology industries - such as Intel, Valve, Tencent and Blizzard - and their prominence eclipses many of the smaller events. Indeed, the high-profile nature of these large events has somewhat focused the idea of esports solely on events with multi-million-dollar prize pools. Yet, as with traditional sports, esports also includes forms of organised play that have no formal tournaments, teams, or prizes, such as collegiate or inter-school competitions, or even impromptu competitions at dedicated club spaces. While these contexts perhaps lack the branded or contractually-determined arrangement with a team as in professional esports contexts, there is a core similarity of having videogame play subject to spectatorship and the idea of play having an audience; specifically of having people watch a videogame without currently playing it. This would seem to cover the variables of esports - videogames played competitively, with spectators.

It is around this core dynamic of competitive and observed play that much of the complicated culture and practices play out, and this is more readily understood and perceived when this occurs over long periods in high-profile spaces such as multi-year tournaments. As with traditional sports, major esports events provide a home for diverse forms of commercialisation. Merchandising, gambling, sponsorship, team memberships, advertising and ticketing are all conventions of esports as much as they are for others. Beyond traditional sports, esports also has something of an unusual approach to tournament prizes. Ticketed events for tournaments for major games such as Dota 2 include the ability for attendees to pay money directly into the tournament purse. The major prize for some tournaments is, consequentially, directly crowdsourced. For Dota 2 this has meant that since 2014 its annual major tournament, "The International", has had purses of over ten million dollars, with the purse increasing year to year. The purse for the International IX in 2019 was USD 34.3 million; for comparison, Wimbledon's purse in 2019 was USD 49.4 million. While tennis and Dota 2 tournaments are perhaps difficult to directly compare, these are still significant sums, especially given that players at the International are as young as 15 and rarely older than 30 (Liquidpedia, 2021). In the context of esports, age is a factor in terms of who plays competitively. It is a matter of common sense within the community that players become too old to play effectively past 24 , as reflexes start to slow down. While the exact reasons for this drop-off are unlikely to be monocausal, it remains the case that esports are aligned with younger players and younger viewers, and it is these younger 
people who have changed their practices of play, viewership and participation during the covid pandemic.

The idea of videogame play being a type of public spectacle has existed for years since the videogame arcades of the 1980s but, beginning in 2007, the shift from these relatively personal contexts to major stadium events has been a significant change. Alongside this, we have seen esports become an industry that sees young people as its primary consumer. And yet it was these physical spaces that were emptied out during the pandemic, with major industry-defining events being cancelled or deferred. The Intel Extreme Masters Season XIV was due to be played in Katowice in 2020 but was deferred to 2021 after much speculation. In 2020, the Overwatch League - a major, publisher-backed league - began competition in February using a multi-city home/away game format that stretched between Asia, North America, and Europe, including the United States, China, South Korea, and France. Players flew between these destinations during February and March, before live events went through a staggered cancellation, leading to the league being unfinished at the end of March when the last live event was cancelled. The tournament would be resolved with online-only play recommencing in July, with finals being resolved in October.

\subsubsection{Who Plays Esports?}

Spectators watch for many reasons (Gibbs et al., 2016) yet, in a survey (n: 888) of people who watch esports, Hamari and Sjöblom (2015) found that the most significant motivation for people to watch esports is because they want to play competitively themselves $(B=0.165, p=0.001)$, with other important motivations being escapism $(B=0.131, p=0.000)$ and enjoyment of aggression $(B=0.117$, $p=0.001)$. Because videogaming by definition requires some digital devices (whether a phone, laptop or full desktop rig), the capacity to play is a commercial activity. While these deals are rarely transparent, a significant part of sponsorship and branding is from companies that sell these devices, or the associated hardware and peripherals (such as webcams, mice/keyboards, headsets, gaming chairs, sports drinks, monitors and graphics cards). Many of these products are sold on the premise of greater skill or endurance; for instance, an industry website describes gaming chairs as helping players to 'sit for longer periods - while gaming at peak performance', noting that 'Esports players spend around 10-12 hours of training at their computers every day' with 'adjustments that let players change position on the fly [keeping] the body in motion while sitting' and enabling players to have 'elite speed, precision, aim, and hand-eye-coordination' necessary for competitive play (ChairsFX, 2021). In this context, the language of professionalisation and elite performance seems to have displaced play as the justification for gaming. Player groups are formalised into branded teams, with 
contractual obligations between players and teams covering things including training commitments, expected performance, player health and wellbeing, specified branded equipment requirements, player behaviour, image rights, sponsor relations, interview expectations and public engagement requirements among other expectations (see sample contract in Taylor, 2012). Players no longer "play", they "train", with their enjoyment being displaced from the narrative of personal enjoyment and replaced with frameworks of success. This distinction, as we elaborate on further below, is important for when participation in esports may fall within the concept of "play" under the CRC.

\subsubsection{Esports Business Models}

It is worth noting that many of the major titles currently played are at least partly free-to-play: League of Legends, Fortnite, Dota 2, cs:Go, Hearthstone, Apex: Legends, and others are available without either a purchase or subscription cost. Even those that require a purchase to play are comparatively cheap. Overwatch, from Blizzard, is USD 19.99; in comparison, major franchise titles such as Red Dead Redemption or Sekiro cost approximately usD 6o on release. The business model of many of these esports-focused titles is different from other games. The emphasis is not on a single purchase, but on a model of periodic spending by players, and should be noted is not exclusive to free-to-play or esports titles. Many of the major tournaments are hosted and run by game publishers, and in some cases, publishers have multiple videogame titles that will have separate tournaments. Blizzard, for instance, runs five separate esports leagues.

The infamous "loot boxes" have been a common model in the past, although loot boxes have been depreciated by many development teams due to concerns about gambling. The model is instructive, however, as it involves players earning boxes of various quality during in-game play which contain random items or skins that are unknown until opened; players then have to purchase keys to unlock these boxes and earn the items. The random reward, which includes items the player may already own, has been the focus of intense criticism from both academic and public contexts, including from the videogame presses, with a common description of the practice as effectively operating as unregulated gambling (Wen et al., 2019). This is only one type of revenue-stream for esports companies and has been somewhat abandoned. Current approaches are much more literal: players simply purchase an in-game currency using "real world" money in a process described as "microtransactions".

Many games operate multiple non-fungible currencies, with one currency earned through play, the other through purchase via credit card. Each currency may have some overlap in terms of what it is spent on, with the earned currency having a more limited range of things that it can purchase. League of 
Legends is perhaps the most visible of the games to make use of this real-world exchange process, but many other games, including some non-esports games, make use of this revenue model. It is easy to see that wealth and available leisure time influence the ability of esports players and viewers to engage with the game. Given that, in many ways, an esports tournament can be understood as a paid advertising experience for both in-game content and for merchandise. This commercialisation of esports, and the interlinked corporate data surveillance practices, may also restrict esports play being self-chosen and free and, accordingly, should be considered when attempting to realise the right to play for children.

\section{3 \\ Digital Play and Children's Development and Enjoyment During COVID-19}

The tension between the CRC's objectives of protecting children from harm and allowing them actively to participate within their own community, especially in situations of crisis (CRC Committee, 2021), is clearly present in the esports environment. Esports offer a digital avenue for children to realise their rights to play, socialise and express themselves at times where offline opportunities are restricted. However, while spending more time and a greater amount of their lived experiences engaged in esports, children are exposed to sophisticated business models and other potentially harmful practices which may lead to them being exploited.

\subsection{Opportunities for the Realisation of the Rights Under Article 31 CRC}

Article 31 explicitly recognises the importance of play and recreation in children's lives, due to its positive impact on the social, cognitive and personal development of the child. Lansdown and Tobin (2019) clarify that this provision is unique in international law and should be understood in a child-centred manner. It is structured around three clusters of rights, which each have their roots in earlier international law instruments. More specifically, States are required to 'recognise the right of the child to rest and leisure, to engage in play and recreational activities appropriate to the age of the child and to participate freely in cultural life and the arts' (Article 31 CRC). Article 31 must be understood holistically, which entails that the different elements all need to be realised (CRC Committee, 2013). A significant question arising from the increased engagement of children in esports during the pandemic is whether, and how, esports can contribute to the realisation of these elements. 
First, the rights to leisure and rest require that children are given unallocated time during which they do not have to study or work or do any other compulsory or enforced activity. During this time, children should be enabled to engage in play and recreational activities. The primary source to draw upon when considering whether esports can be qualified as "play" or "recreational activities" under Article $3^{1}$ CRC, is the definition by the CRC Committee in General Comment No. 17 (2013) on Article 31, which contains a number of key elements. According to this definition, play is, 'any behaviour, activity or process initiated, controlled and structured by children themselves' (Article $3^{1}$ para 1 (c) CRC). The CRC Committee underlines the voluntary nature of play, which is vital to the experience and exercise of the right (Lott, 2020). But while voluntary and consensual participation is important, this is, of course, not determinative for something to be considered play. For instance, certain forms of play by children might involve dangerous, anti-social or violent behaviour while still being consensual.

In addition, Article 31 draws the distinction between "play" and "recreational activities", based on the degree of formality and organisation of the activities. More specifically, "play" can be understood as unstructured informal activities of children uncontrolled by adults, whereas "recreation" refers to more organised and formal activities such as sports or creative arts (David, 2006). What these two types of activities have in common, however, is that they are self-chosen and free. The CRC Committee (2013) underlines that compulsory or enforced games and sports or compulsory involvement in a youth organisation would not qualify as a recreational activity. Play and recreational activities are self-chosen because without active choice and engagement the activity is empty and reduced in meaning and significance. In relation to this, Lott (2020) underlines that 'a child's rights approach to play places children's dignity and agency as central in understanding the right and emphasises its legal protection'. Play has both intrinsic and instrumental value, as it is fun (intrinsic) and involves the exercise of autonomy, physical, mental or emotional activity, thereby expanding children's capabilities (instrumental) (Lott, 2020).

Additionally, the CRC Committee (2013) considers fun, uncertainty, challenge, flexibility and non-productivity as key characteristics of play under the CRC. However, this conception of play has been criticised for focusing primarily on play by younger children and reflecting a lack of understanding of how adolescents play (Lott, 2020). In the esports world, for instance, the characteristics as highlighted by the Committee are highly varied. Many players of esports are contracted and produce wealth for themselves or others during gameplay. Considering that Article 31 awards rights to anyone under the age of 18, the model of play under the CRC should reflect the views of children from 
different age groups, including adolescents. A recent study on child's play and online gaming showed that children (aged 10-16) view the playing of online games as an extension of their offline play activities:

Children say there are benefits to playing online. These can include socialising with their friends, learning new skills, and above all, having fun. In this way, online gaming extends normal play into the digital landscape, and children play by the same social rules that they apply offline.

(CHILDREN'S COMMISSIONER, 2O19)

In light of this, children's engagement in esports could arguably contribute to the realisation of their right to play or recreation, in particular when avenues for offline and outdoor play are restricted.

During the pandemic, playing esports also offered opportunities for children to engage in cultural experiences. For instance, Fortnite introduced live music performances in their gaming environment, attracting a massive number of players (Needleman, 2020). Roblox allowed smaller children to visit virtual theme parks, attend birthday parties and chat to others (Wakefield, 2021). It is widely recognised that new media technologies like social networking, mobile apps and online games, can play an important role in the implementation of Article 31 CRC, by facilitating access to a variety of playful, social, cultural and artistic activities (Hodgkin et al., 2007). Research has shown that gaming knowledge or gaming capital plays a key role in children's broader social structures (Apperley et al., 2013). Participation in popular online games like Fortnite has been found to have an impact on children's social development and forms part of their desire to become less dependent upon parents and family members (Carter et al., 2020). Fortnite is a richly social and creative experience, where players often play in squads, duos or in the four-player "Playground Mode" (i.e. it puts players in control of making their own fun and objectives, thereby allowing for creative play, practice and exploration). The narrative structure that such games adopt mirrors classic event TV content, and as such forms interesting talking points and cultural moments that children can share with their peers. Furthermore, the intersection between online games and other video-sharing or livestreaming platforms such as YouTube or Twitch, allows children to participate in the broader cultural practices originating from the game.

In general, the main obstacles to children's enjoyment of their rights under Article 31 are attitudinal and institutional. More specifically, playful activities are often perceived as less important than other activities like education or work and accordingly receive less attention or priority. In that respect, the 
child's right to play has been labelled the 'forgotten right' in the past (Lott, 2020). A report on the hidden impact of Covid-19 on child rights showed that more than one third of the parents and caregivers reported that their child does not have access to outside space to play, and two in five children reported playing less than before the pandemic (Dulieu et al., 2020). The CRC Committee (2013) encourages States to develop policies and adopt measures needed to enable all children to take full advantage of the opportunities of the digital environment. Such measures include, inter alia, ensuring that children have equal access to the internet and new media technologies and are educated, as well as provided with the necessary skills to use and reap the benefits of such technologies for exercising their right to play. Furthermore, a key requirement for the effective enjoyment of the Article 31 rights is that children engage in play - and recreational activities that are age-appropriate. This is crucial, considering children's tendency to engage in activities they enjoy, but which may be harmful to their development, such as their increasing engagement in sedentary activities like videogaming or the stress experienced by children who voluntarily participate in elite (e)sports (Lansdown et al., 2019).

\subsection{Enabling Play enables Related Rights}

Davey and Lundy underline that Article 31 cannot be looked at in isolation but should be read in conjunction with other key articles in the CRC (Davey et al., 2011). Indeed, Article 31 has an important enabling function for many other rights under the $\mathrm{CRC}$, but especially in relation to the right to freedom of expression (Article 13), freedom of association (Article 15) and educational rights (Articles 28 and 29). As children experience various levels of lockdown conditions during the COVID-19 pandemic, digital spaces for play have become even more important for facilitating connection with others, especially with other children (Gordon, 2021). This part of communication and association between peers - is especially important for realising play. As stated by the CRC Committee (2013), 'children together create forms of imaginative play that are rarely achieved in adult-child relations.' Therefore, enabling play enables the space and environment for speech and association, and simultaneously, facilitating association between peers ensures that children can fully engage in play with other children.

The valuable space that esports create is not only for the players, but for the spectators, as the act of observing or being an audience of esports can give children the opportunity to engage and associate with their peers. However, while esports environments are platforms for speech for young people, the normative boundaries of platforms to be able to control and restrict speech, or indeed the access to games - whether by player sanctions or the architecture of the games 
themselves - is still evolving. For example, in 2019 during a competition hosted by the publisher Blizzard, a player spoke out politically regarding the protests in Hong Kong. Blizzard subsequently banned the player, as well as the commentators at the match, and withheld prize money (Royse, 2019). During the COVID-19 pandemic in 2020, the US Army was criticised for banning viewers who asked about war crimes on its Twitch channel, on the basis that the video game channel is a forum for open debate and accordingly is protected speech in the US (Vincent, 2020). This highlights to some extent the differing jurisdictional legal tests and cultural norms around regulating speech, but also highlights the importance of esports as a space for both speakers and audiences of speech (Barendt, 2016). This importance has dramatically increased during the pandemic given the restrictions on public gatherings. Indeed, the increased time spent on the gaming platforms highlights not only their importance for play but also for the children's capacity to enjoy and exercise key rights such as freedom of expression and assembly in digital spaces. The CRC Committee have made it clear that States have positive obligations to protect the digital environment as a space for seeking, receiving and imparting information, and that children should be protected from 'cyberaggression and threats, censorship, data breaches and digital surveillance' (CRC Committee, 2021). Hence, States have the obligation to ensure that businesses - such as esports platforms - are respecting children's rights (CRC Committee, 2021).

Play has long been associated with children gaining knowledge and skills (Richard, 2017). Accordingly, games, including digital games, have important educational significance. They are used as education tools, as they directly address different forms of learning - cognitive, affective, and physical skills. They can teach values, critical thinking, design thinking etc., ensuring engagement (Richard, 2017). By building on joy and fun, play alleviates stress and allows players to keep immersed in tasks and focused on problem-solving. There is accordingly increasing interest - along with the general rise of educational technologies - to use esports in the educational curriculum (Richard, 2017).

However, although play has these important links with other rights under the CRC, the realisation of Article 31 should not be dependent on it performing its enabling function. In policy terms, this means that play should not only be provided where it appears to achieve some attainable end - such as education or speech or association, but play should be valued in and of itself. Insight here can be gained from Peleg's discussion on the guiding principle of the right to development, in which he calls for acknowledging the child's present and future as equally important (Peleg, 2019). The right to development (and similarly the right to play) is not to be hinged on the future selves of the children. 
The right to play should not only be valued for the role it has in enabling other rights insofar that they are focusing on the child as a future adult (Peleg, 2019), but it should also be valued for its enabling function for children's present enjoyment of the process of development. Esports will likely provide opportunities for children to engage in speech, be exposed to new ideas, associate with others and learn, but the value in this is not the future adults they become. Esports should not only be valued in a formalised sense or if they provide a contribution to an educational curriculum. This again points to the requirement that for esports to be considered "play" or " recreation" under Article 31, they need to be self-chosen by children and free, and uncontrolled by adults. However, while play should be uncontrolled by adults, there is also an obligation to ensure that children are protected from risks and harmful experiences.

\section{The Regulation of Esports and Risk of Exploitative Play}

Under the CRC, States are required to ensure a healthy balance between the promotion of opportunities for play, leisure and culture online with the provision of attractive alternatives for children offline (CRC Committee, 2021). Children's participation and socialisation on esports ecosystems and the benefits for children's rights in times of crisis must, therefore, be finely balanced with their protection from the risks to their rights under Article $31 \mathrm{CRC}$ as well as other rights, including their rights to development (Article $6 \mathrm{CRC}$ ), access to good-quality media (Article 17 CRC), and protection from (economic) exploitation (Article 32 and 36 $\mathrm{CRC}$ ). To find this balance, however, an appreciation of the associated risks to children's rights and the regulatory landscape is required.

\subsection{Exploitation and Exploitative Play during the Pandemic}

Many of the risks emerging from esports ecosystems mirror general concerns surrounding children's participation in the digital world. Children will seek out opportunities to play, even where there are risks to exploitation and other harms present. In focus groups with children and young people, Gordon (2021) found that children sometimes report feeling 'scared' and upset by the content of games or the 'live' interactions with other gamers. As one ten-year-old child stated: 'It [the game] scared the life out of me ... Just eventually like ... I ran ... I ran out of my room ...' (Gordon, 2021). Children also described gaming platforms being used by adults as spaces to approach children in inappropriate ways, with unwanted contact constructing the gaming experience as 'unsafe' for many participants (Gordon, 2021). Without detracting from the 
alarming nature of these risks, sadly they are by no means limited to gaming environments.

A more specific concern that has emerged in both the professional and amateur esports world is the risk of physical and mental exhaustion (Holden et al., 2018). To a certain extent, this concern can be linked to earlier debates about excessive video game consumption and the potential impact on children's health and well-being (e.g. Skoric et al., 20o9), or about overtraining and stress in traditional sports (UNICEF, 2019). In qualitative focus groups, children and young people have reported that they regularly play video games for extended periods of time: 'some ... might be playing the game after school, before school, so first thing in the morning they're waking up to the game', which was viewed as a negative if they missed out on adequate sleep (Gordon, 2021). Further, young people describe the role of parents, guardians and carers as key figures 'at the end of the day say[ing] whether the child is playing in a controlled environment' (Gordon, 2021). These concerns are potentially exacerbated by the dramatic increase in the usage of online gaming platforms during the pandemic described above. Physical and mental exhaustion following excessive consumption of games thus clearly has an impact on children's rights such as the rights to education, and the right to good health, but also the right to play itself as it reduces children's mental capacity and physical time for play (Lott, 2020).

The interactivity that makes esports and streaming so attractive among a young audience also poses other specific challenges. Reference here can be made in relation to so-called "toxic behaviour" exhibited by the gaming community (UNICEF, 2019). This may include racism, homophobia, sexism, hate speech and other forms of cyberbullying, which is partly enabled by anonymity online. CoviD-19 has fuelled racist attacks in video gaming, in particular against Asian players (Kuang, 2020). Children and young people report hearing racist abuse, listening to 'screaming', 'shouting' and 'swearing' during live gaming, which they identify as a negative of engaging with such platforms (Gordon, 2021). They also describe the levels of uncertainty about what they might encounter when using the platforms and adults contacting them posing as other children either directly on the gaming platform or via a second platform such as a social networking site (Gordon, 2021). In this regard, Article $3^{1}$ requires States to ensure that children are protected against such inappropriate activities. Striking the balance between participation and protection is not a straightforward process in digital spaces.

The CRC Committee has emphasised the importance of Article 12 in the digital environment, affirming that, '[w] hen developing legislation, policies, programmes, services and training on children's rights in relation to the digital 
environment, States parties should involve all children, listen to their needs and give due weight to their views' (CRC Committee, 2021). However, children's participation in society goes beyond the right to be heard and incorporates other rights - in particular the right to play in the esports environment - as they can enable and foster children's participation in society. Adopting child-centred and child-informed approaches is one initial starting point. These approaches acknowledge that certain categories of children face additional difficulties in terms of enjoyment and conditions of equality of their Article 31 rights, including girls, children belonging to minorities and children with disabilities (UNICEF, 2019). Heteronormativity and gender stereotyping may constitute a barrier to a balanced and equal access to information communication technologies for children. The branding around an esports team is a merging of the black and fluor-coloured designs of videogame aesthetics with the cuts and sponsorship of traditional sports; alongside this, esports merges the forms of sexism and heteronormativity found in both traditional sports and videogames, leading to predictable outcomes for non-masculine participants despite calls from players for measures to improve this situation (Ruvalcaba et al. 2018; Livingston 2015). For example, ethnographic research on gaming culture found that women and racial minorities are more likely to experience harassment in gaming, effectively limiting participation and socialising within gaming (Richard, 2017). Children are particularly vulnerable to such practices, both as players and as spectators, as they learn by imitation and mimicking what they experience. Therefore, being confronted with heteronormativity, stereotyping and sexism in esports environments can have a significant impact on children's development and how they view themselves. In order to battle gender discrimination in the digital environment, the Council of Europe (2019), for instance, has called on the Member States to take action and promote equality. The providers of esports as well as providers of new media such as gaming livestream platforms or more generally video-sharing platforms may also play an important role here. According to Richard (2013),

female-supportive communities can help mitigate threat by providing counter-stereotypical role models, focusing on training in a supportive environment, and providing resources to understand and manage bias and harassment in game culture.

Furthermore, as children will seek to identify with characters in the game for the enjoyment of their gaming experience, it is important that games offer diversity in characters and their roles. 
Concerns related to the datafication and commercialisation of children's playful activities online also emerge in the context of esports. Children report that the extensive digital surveillance of them and their peer group in esports environments (or even the threat of surveillance) (Gordon, 2021) diminishes the ability of these spaces to be 'free from adult control and management' (Richards, 2017; CRC Committee, 2013). While data collection is driven by corporate interests, the harms from online manipulation can extend further than advertising within esports and may lead to children being 'steered or controlled' in other aspects of their lives (Susser et al., 2019), and as such also impact inter alia, children's rights to privacy (Article $16 \mathrm{CRC}$ ), freedom of thought (Article $14 \mathrm{CRC}$ ), and development (Article $6 \mathrm{CRC}$ ). Hence, while esports may provide space and opportunity for children to learn, communicate and maintain friendships and other social connections, corporate data surveillance practices of online esports may also restrict play being self-chosen and free. In addition, the far-reaching commercialisation of esports can also have a major impact on children's opportunities to enjoy the rights provided for in Article 31 CRC, read together with their right to protection from (economic) exploitation (Article $3^{2}$ and 36 CRC). Back in 2013, the CRC Committee already stressed the major impact of the commercialisation of play on the way children engage in recreation and cultural activities, and urged states to protect children and their families against the pressure they experience from online marketing (CRC Committee, 2013). Lott argues that it 'further reduces the child's access to public space due to the commercialisation and privatisation of such space, and it impacts upon the choice of toys and play for children' (Lot, 2020). Relatedly, parents experience significant pressure to purchase certain games which may be harmful to their children's development (CRC Committee, 2013). The effects of the pandemic, and in particular, the hard lockdown measures, are as of it yet unknown in this regard.

\subsection{A 'Balanced' Children's Rights Response for a Post-Pandemic World}

Considering that children play esports in the private home and digital space, it can be questioned what role the State can play in protecting children from the different kinds of exploitative practices mentioned above. First, while parents have the primary responsibility when it comes to the upbringing and development of their children (Article $18 \mathrm{CRC}$ ), States should aid and support services to parents (CRC Committee, 2021). More specifically,

States parties should regulate and provide guidance for professionals, parents and caregivers and collaborate with digital service providers, as appropriate, to ensure that digital technologies and services intended 
for, accessed by or having an impact on children in their leisure time are designed, distributed and used in ways that enhance children's opportunities for culture, recreation and play. That can include encouraging innovation in digital play and related activities that support children's autonomy, personal development and enjoyment.

(CRC COMMITTEE, 2O21)

Additionally, van der Hof and Groothuis have highlighted that it is the role of the State to encourage children to develop their personality and identity to grow up to become self-reliant and responsible adults (2011). The pandemic forced parents to navigate multiple roles at home, in particular in relation to their children's education.

Second, both the CRC Committee and the Council of Europe have called on States to take up their responsibilities when it comes to establishing legal limits to the monetisation of children's play. More specifically, States should review relevant laws and policies and take measures to ensure children are protected against economic and other forms of exploitation (Council of Europe, 2018). For instance, by introducing data protection safeguards, safety-by-design and other regulatory measures, States should ensure that businesses do not target children using these or other techniques designed to prioritise commercial interests over those of the child (CRC Committee, 2021). Global marketing in this context can also serve to weaken children's active participation in the traditional cultural and artistic life of their community (CRC Committee, 2013). To overcome this, Article 31 requires specific actions of States in the context of marketing and media. More specifically, States are required to 'review their policies concerning the commercialisation of toys and games to children, ... with particular regard to those promoting violence, girls or boys in a sexual way and reinforcing gender and disability stereotypes' (CRC Committee, 2013). However, practically realising such requirements remains challenging.

The very nature of the internet and the profit-making nature of businesses challenge the existing governance framework for online gaming and requires that the various stakeholders involved take up their share of responsibility (including legislators, policymakers, game developers and parents) (IGF, 2020). However, while there may be challenges that are different to traditional sports and play, Thierer cautions us on responding to "technopanics" with regulation by prohibition. He considers technopanics similar to "moral panics" in which there is an 'intense public, political, and academic response to the emergence or use of media or technologies, especially by the young' (Thierer, 2014). He highlights that moral panics, technopanics and youth protection rationales have been used to justify information control from comic books to video 
games. It is important that responses to legal challenges of esports are not met with an overly protectionist approach - based on regulatory methods such as parental control or screen time limits - to the detriment of children's participation in esports, and correspondingly their participation in play. For instance, the WHO's inclusion of a gaming disorder in the International Classification of Diseases has been criticised for potentially leading to undue restrictions of children's agency and the rights to play and freedom of expression. It might also lead to children who play games and identify as gamers feeling increasingly stigmatised by their parents, schools or communities (UNICEF, 2019).

Finding activities that children can do from home safely during lockdown and quarantine has been a main priority for many parents. In relation to this, States can require guidance from esports providers in the form of age ratings or labelling mechanisms. When doing so, they should be formulated in a way that does not curtail children's access to the digital environment as a whole or overtly restrict the opportunities for their rights under Article 31 CRC (CRC Committee, 2021). The principle of the evolving capacities of the child requires that once children are competent to exercise their rights, they should be able to do so. In practice, this means that when children become increasingly competent, parents have to fulfil fewer parental responsibilities (Reynaert et al., 2009) and states should consider this principle when establishing age restrictions (Lievens et al., 2018). The balancing act between children's capacity to exercise their rights and their relative lack of experience is also a necessary exercise in the context of esports. From a certain age, they will be better equipped to understand the potential risks emerging from engaging in esports and livestreaming and therefore better equipped to exercise their rights.

Moreover, it has been argued that research on children and online gaming, and esports conducted so far, is not robust or reliable enough to inform policy decisions or best practice recommendations (UNICEF, 2019). This reflects the clear need for a stronger evidence-base for policy making. The CRC Committee (2013) also recognises that while children do need to be protected from harm in the realisation of their rights under Article 31 CRC, some degree of risk and challenge is integral to play and recreational activities and can be considered a necessary element of the benefits of these activities. Hence, there is a need to find a balance between taking action to reduce unacceptable risks to exploitation and harm arising from the esports world on the one hand, and on the other hand, educating children to become 'gaming literate' and experience empowered online play.

Finding such a balance between children's play and protection is, of course, not an easy task. When determining the level of risk to which children can be exposed, the best interests of the child and children's own 
views and experiences should be guiding principles (CRC Committee, 2013). Children often have exceptional knowledge about online gaming environments and how it affects them and their rights. Hence, policy makers should allow them actively to contribute to knowledge creation on how their rights should be conceptualised and operationalised in this specific context, in line with Article 12 CRC (Hanson et al., 2020). Children themselves have suggested that the gaming industry should have youth panels which would ensure that the design of platforms, games and complaints procedures are accessible and made much safer as spaces for children (Gordon, 2021). This is similar to the "safety by design" approach advocated for by the e-safety Commissioner in Australia.

However, other than as research subjects, children are rarely consulted in studies on gaming-related harms, nor are they consulted on policies to restrict gaming activities (UNICEF, 2019). There is a clear need for adults to understand children's fears and concerns and also for children to have input into the strategies implemented by adults and institutions to deal with such concerns. The facilitation of an environment in which children can express their concerns will allow for the better identification of potential harms and development of child-centred ways of responding to them in a manner that is cognisant of children's best interests. There is a need for more research to determine how children's voices can be properly integrated to respect their right to be heard and to gain insights into their perceptions of play, participation, and the risks associated with the esports phenomenon. The pandemic has exacerbated this need.

The increased engagement of children in the esports world raises a number of concerns from a children's rights perspective. They potentially both positively enable, but also negatively impact, the child's right to play and related rights simultaneously. However, the complexity of the gaming ecosystem and the underlying business models render the extraction of generalisable insights challenging. A more nuanced, context dependent approach which recognises that the term "esports" comprises both large-scale commercial events and small-scale play between friends, is therefore required. The covid-19 pandemic has clearly demonstrated the importance of gaming for children in terms of their socialisation, and engagement in cultural and playful experiences and thus, a broad-brush approach may fail to recognise the benefits of such gaming ecosystems. 
"Esports" as a category may be too diverse to attract general regulatory interventions. That being said, this does not exclude the more targeted regulation of theunderlying risks discussed above. Moreover, although certain features underpinning esports may require new approaches to child safety and well-being, any such regulatory responses should not lose sight of the need for avenues for children to participate in the (online) community and exercise their rights, including their right to play, freedom of expression and development. This is particularly important when one considers the increased use and thus significance of gaming platforms for children during the pandemic. More research is required to determine how this might be achieved in practice, including insights into children's own views on playing different types of esports and their rights in this particular context. There is a need for a balanced regulatory approach, which incorporates the guiding principles of the CRC of taking into account children's best interests, their right to be heard, their right to development, and ensuring that children are afforded their rights without discrimination. This article has aimed to set the stage for this more detailed research, which places children's voices at the centre.

\section{References}

Abarbanel, B., "Gambling vs. gaming: A commentary on the role of regulatory, industry, and community stakeholders in the loot box debate", Gaming Law Review 22.4, 2018, 231-234.

Apperley, T. and Leorke, D., "From the cybercafé to the street: the right to play in the city", First Monday 2013 (18 (11).

Barendt, E., Freedom of Speech, 2nd edn. (Oxford: Oxford University Press, 2016).

Businesswire, "Games Industry Unites to Promote World Health Organization Messages Against Covid-19; Launch \#PlayApartTogether Campaign", 2020: https://www. businesswire.com/news/home/20200328005018/en/Games-Industry-UnitesPromote-World-Health-Organization.

Carter, M., Moore, K., Mavoa, J., Horst, H. and Gaspard, L., "Situating the Appeal of Fortnite Within Children's Changing Play Cultures", Games and Culture 2020 (15), 453-471.

Chairs FX, 2021: https://chairsfx.com/buying-guides/pro-esports-chairs/.

Children's Commissioner, Gaming the system, 2019: https://www.revealingreality. co.uk/wp-content/uploads/2019/10/cco-gaming-the-system.pdf.

Council of Europe, Recommendation CM/Rec(2018), on Guidelines to respect, protect and fulfil the rights of the child in the digital environment, 4July 2018. 
Council of Europe, Recommendation $\mathrm{CM} / \operatorname{Rec}(2019)$, Preventing and Combating Sexism, 27 March 2019: https://rm.coe.int/prems-o55519-gbr-2573-cmrec-2019-1web-a5/168o93eo8c.

CRC Committee, General Comment No. 25 (2021) on Children's Rights in relation to The Digital Environment.

CRC Committee, General comment No. 17 (2013) on the riht of the child to rest, leisure, play, recreational activities, cultural life and the arts (Art. 31).

Davey, C. and Lundy, L., "Towards Greater Recognition of the Right to Play: An Analysis of Article 31 of the UNCRC", Children \& Society 2011 (25), 3-14.

David, P., A Commentary on the United Nations Convention on the Rights of the Child, Article 37: The Right to Leisure, Play and Culture (Leiden: Martinus Nijhof, 2006).

Dulieu, N. and Burgess, M., "The Hidden Impact of Covid-19 on Child Rights" (London, Save the Children International, 2020).

ESafety Commissioner, The digital lives of Aussie teens, 2021: https://www.esafety.gov. $\mathrm{au} /$ sites/default/files/2021-02/The\%2odigital\%2olives\%2Oof\%2OAussie\%2oteens. pdf.

Escharts.com, 2019: https://escharts.com/tournaments/iem-katowice-2019.

ESIC, The Importance of Child Protection in esports, 2019: https://esic.gg/theimportance-of-child-protection-in-esports/\#.

Gibbs, M., Carter, M., Cumming, D., Fordyce, R. and Witkowski, E., "Esports Spectatorship in Australia", Networked Society Institute Research Paper, Melbourne: University of Melbourne, 2018: https://emmawitkowski.files.wordpress.com/2018/og/esportsaustralia-nsi_gibbs_carter_cumming_fordyce_witkowski_2018.pdf.

Gordon, F., Online Harms Experienced by Children and Young People: 'Acceptable Use' and Regulation (London: Catch22, 2021, forthcoming).

Hamari, J. Sjöblom, M., "What Is eSports and Why Do People Watch It?", Social Science Research Network, 2015, 1-26. Doi.org/10.1108/IntR-04-2016-0085.

Hanson K. and Nieuwenhuys O., Reconceptualizing Children's Rights in International Development : Living Rights, Social Justice, Translations (Cambridge Univ Press 2013) https://archive-ouverte.unige.ch/unige:91151.

Hodgkin, R. and Newell, P., Implementation Handbook for the Convention on the Rights of the Child: Fully Revised Third Edition (UNICEF, 2007).

Holden, J., Kaburakis, A. and Rodenberg, R., "Esports: Children, stimulants and videogaming-induced activity", Journal of Paediatrics and Child Health, 2018.

I G F 2020, Online Gaming and Child Rights: https://www.intgovforum.org/multilingual/ content/igf-202O-ws-53-right-to-play-online-gaming-and-child-rights\#undefined.

Kuang, W., "Pandemic fuels a virulent new strain of racist attacks in online gaming", The Citizen,2020:https://www.thecitizen.org.au/articles/pandemic-fuels-a-virulentnew-strain-of-racist-attacks-in-online-gaming. 
Lansdown, G. and Tobin, J., "Article 31: The Rights to Rest, Leisure, and Play", in The UN Convention on the Rights of the Child - A Commentary (Oxford: Oxford University Press, 2019).

Lievens, E., Livingstone, S., McLaughlin, S., O’Neill, B. and Verdoodt, V., "Children's rights and digital technologies", in U. Kilkelly , and T. Liefaard (eds.), International Human Rights of Children (Singapore: Springer, 2018).

Liquidpedia, Player Statistics Portal. 2021: https://iquipedia.net/leagueoflegends/ Portal:Statistics

Livingston, C., GDC panel: women in esports are "walking through a minefield", 2015: https://www.pcgamer.com/gdc-panel-women-in-esports-are-walking-through-aminefield/.

Livingstone, S., Byrne, J., Carr, J., “One in Three: Internet Governance and Children's Rights”, Innocenti Discussion Paper No.2016-o1, UNICEF Office of Research Florence 2016, $1-36$.

Liquidpedia, Player Statistics Portal. 2021: https:/liquipedia.net/leagueoflegends/ Portal:Statistics

Lott, N., "The Right to Play", Thesis submitted to The University of Nottingham for the degree of Doctor of Philosophy, 2020.

Lopez-Cabarcos, M. A., Ribeiro-Soriano, D. and Pineiro-Chousa, J., "All that glitters is not gold. The rise of gaming in the CoviD-19 pandemic", Journal of Innovation \& Knowledge 2020 (5), 289-296.

Needleman, S., "Welcome to 'Fortnite' - Enjoy the Concert”, The Wall StreetJournal, 2O2O: https://www.wsj.com/articles/welcome-to-fortniteenjoy-the-concert-11593004563.

oECD, PISA 2015 Results (Volume III): Students' Well-Being (Paris: oEcD Publishing, 2017).

Peleg, N., The Child's Right to Development (Cambridge: Cambridge University Press, 2019).

Reynaert, D., Bouverne-de-Bie, M. and Vandevelde, S., "A Review of Children's Rights Literature Since the Adoption of the United Nations Convention on the Rights of the Child", Childhood 2009 (16), 518.

Richard, G., "Video Games, Gender, Diversity, and Learning as Cultural Practice: Implications for Equitable Learning and Computing Participation Through Games", Educational Technology, March-April 2017 (57(2)), 3-43.

Royse, D., Blizzard Games Caught Up In Free Speech Question Over Hong Kong Protests, Bans Gamer Over Remarks, 2019: https:/www.benzinga.com/news/19/10/14563449/ blizzard-games-caught-up-in-free-speech-question-over-hong-kong-protests-bansgamer-over-remarks.

Ruvalcaba, R., Shulze, J., Kim, A., Berzenski, S. R. and Otten, M. P., "Women's Experiences in eSports: Gendered Differences in Peer and Spectator Feedback During Competitive Video Game Play", Games and Culture, 2018. 
Skoric, M., Teo, L. and Neo, R., "Children and Video Games: Addiction, Engagement, and Scholastic Achievement”, Cyberpsychology \& behavior 12(5)., 20o9, 567-72. Doi: 10.1089/cpb.2009.0079.

Smahel, D., Machackova, H., Mascheroni, G., Dedkova, L., Staksrud, E., Ólafsson, K., Livingstone, S. and Hasebrink, U., "EU Kids Online 2020: Survey results from 19 countries", EU Kids Online, 2020: Doi.org/10.21953/lse.47fdeqjo1ofo.

StreamElements, Report - State of the Stream December/ 2020 Year in Review, 2021, StreamElements.com.

Susser, D., Roessler, B. and Nissenbaum, H., "Technology, autonomy, and manipulation”, Internet Policy Review, 8(2), 2019, Doi: 10.14763/2019.2.1410.

Sznajder, K., Katowice 2019 recap: record breaking attendance and online viewership, 2019: https://musecasino.com/news/katowice-2019-new-viewership-records/.

Taylor, T. L., Raising the Stakes - E-Sports and the Professionalization of Computer Gaming (Boston: MIT Press, 2012).

Thierer, A., "A Framework for Responding to Online Safety Risks", in Minding Minors Wandering the Web: Regulating Online Child Safety (The Hague: T.M.C. Asser Press, 2014).

Third, A., Bellerose, D., Dawkins, U., Keltie, E. and Pihl, K., Children's Rights in the Digital Age [Documento Elettronico]: a Download from Children around the World (Young and Well Cooperative Research Centre, 2014).

UNICEF, Child rights and online gaming: opportunities and challenges for children and the industry, 2019: https://www.unicef-irc.org/files/upload/documents/UNICEF_ CRBDigitalWorldSeriesOnline_Gaming.pdf.

UN News, UN chief calls for greater protection for children caught up in COVID-19 crisis, 2020: https://news.un.org/en/story/2020/o4/1061892.

van der Hof, S. and M. Groothuis M. (eds.), Innovating Government, Normative, policy and technological dimensions of modern government (The Hague: T. M. C. Asser, Berlin/Heidelberg: Springer, 2011).

Verdoodt, V., Clifford, D. and Lievens, E., “Toying with children's emotions, the new game in town?", Computer Law and Security Review, 2016, 599-614.

Verizon, Verizon Network Report, reflecting 9 March 9 compared tor6 March 2020, 2020: https://www.verizon.com/about/news/how-americans-are-spendingtheir-time-temporary-new-normal.

Vincent, J., US Army pauses video game streams after accusations of violating free speech, 2020: https://www.theverge.com/2020/7/23/21335347/us-army-militarypauses-esports-twitch-streaming-war-crimes-criticism.

Wakefield, J. "Roblox: How the children's game became a \$3obn bet on the Metaverse", 2021: https://www.bbc.com/news/technology-56345586.

Wen, L., Mills, D. and Nower, L., "The relationship of loot box purchases to problem video gaming and problem gambling", Addictive behaviors 97, 2019, 27-34. 
WHo, International Statistical Classification of Diseases and Related Health Problems (ICD-11), 2019: https://www.who.int/standards/classifications/classification-ofdiseases.

Zendle, D., "Beyond loot boxes: a variety of gambling-like practices in video games are linked to both problem gambling and disordered gaming", PeerJ 8, 2020. Doi. org/10.7717/peerj.9466.

Zosh, J. M., Hopkins, E. J., Jensen, H., Liu, C., Neale, D., Hirsh-Pasek, K., Solis, L. and Whitebread, D., Learning through play: A review of the evidence (White Paper) (Billund: The LEGo Foundation, 2017). 